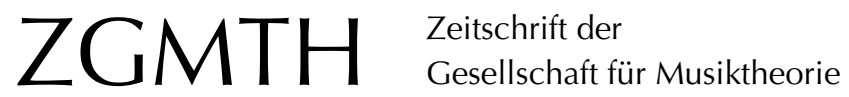

Schäfer-Lembeck, Hans-Ulrich (2011): Musik machen, denken, kommunizieren. Überlegungen, ausgehend von den Fragen zum Verhältnis von Musikpädagogik und Musiktheorie. ZGMTH 8/1, 37-38. https://doi.org/10.31751/615

() 2011 Hans-Ulrich Schäfer-Lembeck

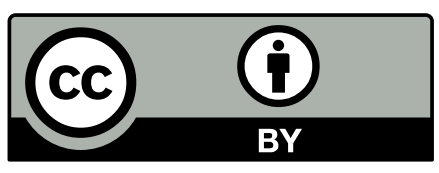

Dieser Text erscheint im Open Access und ist lizenziert unter einer Creative Commons Namensnennung 4.0 International Lizenz.

This is an open access article licensed under a Creative Commons Attribution 4.0 International License.

veröffentlicht / first published: 14/06/2011

zuletzt geändert / last updated: 23/07/2012 


\section{Musik machen, denken, kommunizieren \\ Überlegungen, ausgehend von den Fragen zum Verhältnis von Musikpädagogik und Musiktheorie}

Hans-Ulrich Schäfer-Lembeck

Mit Musiktheorie kann ein Denken und Reden über Musik mit Reichweiten wie in subjektiven oder Alltagstheorien genauso bezeichnet sein wie eine wissenschaftliche Theorie, die demgegenüber einen höheren Grad an Bewusstheit, ausdrücklicher Formulierung und die Einbeziehung von systematischer Beobachtung hat.

Wenn ich gefragt werde, wie ich zu meinem Verhältnis zur Musiktheorie gekommen bin, will ich vermutend-erklärend eine Erinnerung an meine Studienzeit anführen. Eingebunden in ein von Achtung, Engagement und Interesse geprägtes Arbeitsklima ging es dort in der Arbeit im Fach Musiktheorie um historisch differenzierendes Auffassen und Beschreiben von Musik. Es ging um Musik als Produkt von Denken und um, wie der Dozent es nannte, "Kunst als Medium der Ungeborgenheit«, wobei die satz- und klavierpraktische Arbeit durchgängig ein wichtiger Bestandteil war. Manche Ideen und Formulierungen aus diesem Unterricht nutze ich inzwischen verändert, eine grundsätzliche Freude aber, mich näher mit Musik (auch strukturell) zu beschäftigen, hat sich prinzipiell erhalten. Und dieser Unterricht, das dort angestoßene Lernen war so, dass es auch zu fachlichen Anwendungen erquicklicher und erfolgreicher Art geführt hat, die in dieser Form nicht direktes Unterrichtsthema gewesen waren; es war also um musikalische und musikbezogene Kompetenz in einem umfassenderen Sinne gegangen.

Musiktheorie gehört in ein Schulmusikstudium, damit Studierende, zukünftige Musiklehrkräfte, satzpraktisch handlungsfähig werden und wissenschaftlich eingebettetes Denken in und über die Strukturen von Musik handhaben können. So kann hier die Bildung von sensiblen, wachen, klugen Persönlichkeiten mitangestoßen werden, die zum Agieren und zu Perspektivwechseln in der Lage sind. Das Musiklehramtsstudium enthält ein gerüttelt $M a ß$ an musikpraktischen Anteilen, die wie ein Handwerk im Meister-Lehrling-Prinzip vermittelt werden, primär auf Funktionieren und Anwendung gerichtet sind; Kunst wird hier oft als irrationales Metier gepflegt, in dem vorzugsweise im egomanischen Habitus agiert wird. Demgegenüber zielt Wissenschaftlichkeit auf eine andere Qualität von Umgehensweisen; ihr geht es darum, in Diskurse eingebettet, Gegenstände, Blickwinkel und Ergebnisse zu reflektieren und eben auch zu relativieren. Lehrerbildung erfordert Wissenschaftlichkeit, weil in Erziehung und Bildung aufgrund zukünftiger schneller und unvorhersehbarer Entwicklungen auf große Veränderungen zu reagieren sein wird. Dazu ist die Befähigung, bisherige Sichtweisen auf- und neue zu er- 
arbeiten erforderlich, weil rezeptologisches Anwendungswissen (worauf Handwerk und Ausbildung zielen) veraltet und so keine geeignete Grundlage für verantwortliches Handeln bietet (deswegen auch: Lehrerbildung und nicht: Ausbildung!). Guter Unterricht an Schulen bedarf lehrerseitig theoriegeleiteter, methodenbewusster, kritisch-unabhängiger, weitgefasster Kompetenz. Diese umfasst wissenschaftliche Überlegungen zu den Voraussetzungen und Bedingungen musikalischen Lernens, zu dessen institutionellen und unterrichtlichen Praxen genauso wie zur Geschichte und zu den Strukturen von Musik. Insofern sollte meines Erachtens das Fach Musiktheorie Studierende befähigen, Musik strukturell, in ihrer Gemachtheit wahrnehmen, erforschen und beschreiben zu können. Solches Beschreiben heißt Interpretieren und heißt etwas Erscheinendes nicht für bare Münze zu halten, sondern begründend Bedeutungen zuzuweisen, Entscheidungen zu treffen bzw. solche zu erwägen und gegebenenfalls weiterzuentwickeln. Dabei muss meines Erachtens nicht allein begrifflich, sondern auch praktisch gearbeitet werden.

Musiktheorie sollte es auch in der Schule geben, im Unterricht, weil auch das verstandesmäßige Umgehen mit Musik eine Praxis ist, die in der bürgerlichen Gesellschaft allen zusteht und kein Privileg von wenigen ist. - Um in der sensiblen und klugen Beschäftigung mit Musik (jedenfalls mittelfristig) über Denkrezepte oder bloß subjektive Theorien hinauszukommen, sind im Gespräch abgeglichene bzw. in intersubjektiven Diskursen verortete und geordnete Begriffe und Vorstellungen unentbehrlich. Bildung ermöglichen (auch auf Musik und deren Strukturen bezogene) hieße also die Möglichkeit der Partizipation an derartigen Diskursen schaffen. Dabei ist nicht allein die Sachseite, sondern auch die Beziehungsseite zu beachten und zu pflegen.

Das Verhältnis der Disziplinen Musikpädagogik und Musiktheorie in der Hochschullandschaft hierzulande ist verschieden. Dort, wo Vertreter der Musikpädagogik ihre besondere Zuständigkeit für Schulmusik als Inhaber von Richtlinienkompetenz leben oder Vertreter der Musiktheorie als Inhaber musikbezogener Deutungshoheit, ist oft auch die Beziehung der Exponenten und produktives Miteinander schwierig. Dort, wo man miteinander arbeitet und die gemeinsamen und unterschiedlichen Zuständigkeiten sortiert, gibt es Beispiele für ein sehr gutes Verhältnis. Dann können Gemeinsamkeiten bearbeitbar werden, z. B. wenn Musikpädagogik und Musiktheorie als operativ aufgefasste Wissenschaften in den fachlichen und systemischen Biotopen von Institutionen auf ganz ähnliche Widerstände stoßen. - Allgemein würde ich sagen, dass das Verhältnis zwischen den beiden Disziplinen in vielen Fällen von genauso viel Mangel an Kenntnis voneinander und Respekt füreinander gekennzeichnet ist wie zwischen anderen Hochschuldisziplinen auch. Weiterentwicklungsmöglichkeiten in Sachen Achtsamkeit auf Andere und Anderes - in Hinsicht auf Kommunikationskultur - gibt es hier nicht speziell. 